\title{
ON THE MAXIMUM DEFICIENCY OF $r$-SPACE CURVES
}

\author{
BY B. C. WONG
}

The problem of determining the greatest deficiency of an $r$-space curve of given order seems yet unsolved. This problem is equivalent to that of determining the maximum dimension of a sub-space of $S_{r}$ in which a curve of given order and genus can lie. Thus the maximum genus of a 4 -space sextic curve is 2 ; but if the sextic curve is of genus 3 or 4 , it is necessarily a 3-space curve or a plane curve; and if it is of genus greater than 4 , it is a plane curve.

It is well known that the deficiency of an $r$-space curve $C^{n}$ of order $r$ is zero. Veronese* has shown that the greatest deficiency of an $r$-space curve $C^{r+s}$ where $s<r$ is $s$ and that of $C^{2 r}$ is $r+1$. It is the purpose of this paper to present a method of calculating the maximum deficiency of curves whose orders are greater than $2 r$.

To determine the greatest deficiency $P$ of a curve $C^{n}$, it is only necessary to determine the least number, $h$, of apparent double points of its projection on an $S_{3}$, for

$$
p=(n-1)(n-2) / 2-h .
$$

We shall use the phrase the apparent double points of $C^{n}$ instead of the apparent double points of the 3-space projection of $C^{n}$. To determine $h$, we make use of the formula

$$
\left.D=\mu_{1} \mu_{2} \cdots \mu_{r-1}\left(\mu_{1} \mu_{2} \cdots \mu_{r-1}-\sum \mu_{i}+r-2\right) / 2\right),
$$

for the number $D$ of apparent double points of $C^{n}$ which is the complete intersection of $r-1$ hypersurfaces of order

* Behandlung der projectivischen Verhältnisse der Räume von verschiedenen Dimensionen durch das Princip des Projicirens und Schneidens, Mathematische Annalen, vol. 19 (1882), pp. 161-234.

† Loc. cit., p. 205. 
$\mu_{1}, \mu_{2}, \cdots, \mu_{r-1} . D$ will be a minimum if $r-2$ of the hypersurfaces are hyperquadrics. Hence, putting

$$
\mu_{1}=\mu_{2}=\cdots=\mu_{r-2}=2, \quad \mu_{r-1}=n / 2^{r-2},
$$

in (2), we have

$$
h \geqq \frac{n}{2^{r-1}}\left[\left(2^{r-2}-1\right) n-2^{r-2}(r-2)\right],
$$

and

$$
p \leqq \frac{1}{2^{r-1}}\left[n^{2}+2^{r-2}(r-5) n+2^{r-1}\right]
$$

The equality signs hold when $n=2^{r-2} \mu_{r-1}$, where $\mu_{r-1}>1$. When $n \neq 2^{r-2} \mu_{r-1}$, (3) gives too low a limit for $h$ and (4) gives too high a limit for $P$. To find the exact value of $h$ we distinguish two cases: $n$ even and $n$ odd.

First take the case $n$ even and equal to $2 m$. Consider an equivalent degenerate curve $C^{2 m}$ made up of two $(r-1)$-space curves $C^{m}$ and $C^{\prime m}$ lying in different $(r-1)$-spaces but having $m$ points in common. If $h^{\prime}$ is the least number of apparent double points on each of $C^{m}$ and $C^{\prime m}$, then

$$
h=m^{2}-m+2 h^{\prime},
$$

since $m^{2}-m$ is the number of apparent intersections of $C^{m}$ and $C^{\prime m}$. Replacing $m$ by $n / 2$ in the above, we have

$$
h=n(n-2) / 4+2 h^{\prime}
$$

and

$$
p=(n-2)^{2} / 4-2 h^{\prime} \text {. }
$$

Now take the case $n$ odd and equal to $2 m-1$. Consider an equivalent degenerate curve $C^{2 m-1}$ composed of two $(r-1)$ space curves $C^{m}$ and $C^{m-1}$ lying in different $(r-1)$-spaces but having $m-1$ points in common. Letting $h^{\prime \prime}$ be the least number of apparent double points on $C^{m}$ and $h^{\prime \prime \prime}$ be that of apparent double points on $C^{m-1}$, we have

$$
h=(m-1)^{2}+h^{\prime \prime}+h^{\prime \prime \prime},
$$


since $(m-1)^{2}$ is the number of apparent intersections of $C^{m}$ and $C^{m-1}$. If we replace $m$ by $(n+1) / 2$, the result is

$$
h=(n-1)^{2} / 4+h^{\prime \prime}+h^{\prime \prime \prime}
$$

and hence

$$
P=(n-1)(n-3) / 4-h^{\prime \prime}-h^{\prime \prime \prime} .
$$

To find $h^{\prime}$ or $h^{\prime \prime}$ and $h^{\prime \prime \prime}$ we repeat the process. We soon arrive at the component curves whose orders are equal to or less than twice the dimensions of the several sub-spaces in which the component curves lie, and then apply Veronese's rule. Or, in case $n$ is very large, we arrive at the component curves which are all plane and have no double points.

It is to be noticed that $h^{\prime}=h^{\prime \prime}=h^{\prime \prime \prime}=0$ if $r=3$. Hence the greatest deficiency of any 3 -space curve $C^{n}$ is

and

$$
p=(n-2)^{2} / 4, \quad \text { for } n \text { even; }
$$

$$
p=(n-1)(n-3) / 4, \quad \text { for } n \text { odd. }
$$

The University of California

\section{A NOTE ON CERTAIN CONTINUOUS NON-DIFFERENTIABLE FUNCTIONS*}

BY F. W. PERKINS

This note gives a treatment of some phases of the theory of a class of functions of which a particular example has already been studied by the author in a note entitled $A n$ elementary example of a continuous non-differentiable function, in the American Mathematical Monthly (vol. 34 (1927), pp. 476-478). The method there used for the construction of a function with the desired properties bears some resemblance to that used by Brodén, Köpke, and Steinitz for the

* Presented to the Society, September 6, 1928. 